Anja Kurzhals *, Christoph Brandt-Wunderlich, Niels Grabow, Klaus-Peter Schmitz, Wolfram Schmidt

\title{
Dynamic image analysis of transparent particles released during the simulated use test of cardiovascular devices
}

\begin{abstract}
For product approval of coated cardiovascular devices, the assessment of particle release is essential. Particularly challenging are delivery systems equipped with hydrophilic coatings releasing highly transparent particles. The aim of this study was to compare two different methods of particle counting, namely the light obscuration method and the dynamic image analysis (DIA), with respect to optical transparent particles.

The particles were collected during the simulated use of cardiovascular catheters and analysed in suspension with a dynamic imaging device (FlowCam, Fluid Imaging Technologies). Particles were detected by a greyscale threshold and imaged to analyse their shape and transparency. The statistical influence of the threshold on particle counts and size distribution was determined and compared to light obscuration particle counting (Model 9703 with sensor HRLD 400CE, HIAC ROYCO).

The light obscuration method provided lower particle counts in suspensions containing a high amount of transparent particles. The lower the detection threshold, the higher the particle counts were. In conclusion, it is important to adapt the threshold value for samples that are expected to contain a high amount of transparent particles. DIA may be suggested as a valuable additional method for particulate analysis.
\end{abstract}

\footnotetext{
*Corresponding author: Anja Kurzhals: Institute for Biomedical Engineering, Rostock University Medical Center, RostockWarnemuende, Germany, e-mail: anja.kurzhals@uni-rostock.de Christoph Brandt-Wunderlich: Institute for ImplantTechnology and Biomaterials e.V., Rostock-Warnemuende, Germany, e-mail: christoph.brandt-wunderlich@uni-rostock.de Niels Grabow, , Wolfram Schmidt: Institute for Biomedical Engineering, Rostock University Medical Center, RostockWarnemuende, Germany, e-mail: niels.grabow@uni-rostock.de, wolfram.schmidt@uni-rostock.de

Klaus-Peter Schmitz: Institute for ImplantTechnology and Biomaterials e.V. and Institute for Biomedical Engineering, Rostock University Medical Center, Rostock-Warnemuende, Germany, e-mail: klaus-peter.schmitz@uni-rostock.de
}

Keywords: particle analysis, cardiovascular implants, dynamic image analysis, hydrophilic coating

https://doi.org/10.1515/cdbme-2019-0052

\section{Introduction}

Cardiovascular implants such as drug coated balloons (DCB) or drug eluting stents (DES) are used to treat coronary artery disease. The approval of those implants includes the analysis of particulate matter, because particles released into the blood stream can increase the risk of emboli [1]. The investigation of the particle release of coated devices is necessary according to guidance of the authorities $[2,3]$. Sub-visible particles might originate from the manufacturing process, abrasion or delamination during implantation. In this study the focus is set on particles emitted from devices that have a hydrophilic coating. These coatings are used to avoid friction and damages of the vessel wall during the implantation process [1]

The particles were collected during the simulated use of cardiovascular catheters and analysed in suspension. A standard test method to count the particles and to measure the particle size distribution is the light obscuration method (LOM) with regard to the USP788 [4]. To visualize the particles directly in suspension the samples were investigated additionally with dynamic image analysis (DIA). The aim of the study was to test the sensitivity of both methods for the detection and the size determination of transparent particles. 


\section{Material and methods}

\subsection{Simulated Use Procedure}

Particle solutions were generated during a simulated use procedure of balloon catheters. Therefore, three balloon catheters were advanced through a tortuous path according to ASTM F2394-07(2017) [5], dilated into a silicone mock vessel, deflated and withdrawn from the test path. To collect the particles generated during the procedure, particle free water was flushed through the test past and collected in a clean vial.

The test samples contained the same hydrophilic coating. All tests were done under clean room conditions. The volume of the test suspension contained a volume of $25 \mathrm{ml}$.

\subsection{Particle counting}

Particle counting was done with a particle counter based on the LOM (HIAC Royco 9703, sensor model HRLD400, $\mathrm{HACH}$, Loveland, Colorado, USA). For each sample a volume of $20 \mathrm{ml}$ was tested and the samples were homogenized. [4] For the study particles between 10 and $500 \mu \mathrm{m}$ were considered.

Therefore, the particle suspension sample was primed into a cuvette that is illuminated with a laser beam. A detector measured the brightness on the opposite side of the cuvette. Every particle that passes the optical sensor causes a change in the brightness and, in consequence, a pulse in the electrical signal. Every pulse is counted and the number of pulses equals the number of counted particles. The particle size is calculated from the pulse width [6]. The conversion factor between the pulse width and the particle size was calibrated with spherical standard particles.

\subsection{Dynamic image analysis}

The DIA was done with the FlowCam 8000 (FluidImaging, Scarborough, Maine, USA). For the imaging, a volume of $4 \mathrm{ml}$ of the particle suspension was directed through a transparent flow cell (Field of View $300 \mu \mathrm{m}$ ) that was illuminated with a LED Flash Lamp. Images of the whole flow cell were taken with a CCD sensor on the opposite side of the illumination with a frame rate of 29 images per second. The images were saved and analysed with the Software VisualSpreadsheet Version 4.12.3 (FluidImaging). To identify the particles the images of the flow cell were converted into binary images. Every pixel with a higher or lower greyscale than the mean value of the background is identified as a pixel belonging to a particle. The identification of particles should always include darker and brighter pixels [7]. Adjacent pixels are allocated to the same particle image. The difference between the greyscale of pixels belonging to a particle and the pixels rated as background pixels is introduced as a so-called threshold. After the identification of the particles in the image, particle images are cropped and listed in a view chart. Binary images can be overlaid to control the fitting of the digital image analysis.

With the help of the binary images the particle size is calculated. The conversion factor between pixel and micrometre was calibrated with spherical polystyrene beads. The chosen method to determine the diameter of a particle is the equivalent sphere diameter (ESD). The mean value of 36 Feret diameters in different angles around the particles is taken as the diameter [8]. Particles between 10 and $500 \mu \mathrm{m}$ were considered. The threshold value was varied between 10 and 30 to validate the influence on the particle sizing.

\section{Results}

\subsection{Particle counting with light obscuration}

The results of the particle counting and sizing with the LOM are shown in table 1 . The counts were calculated as particle concentration in particles per $\mathrm{ml}$.

Table 1: Particle concentration in particles $/ \mathrm{ml}$ counted with LOM

\begin{tabular}{rrrr}
\hline Particle size & Catheter 01 & Catheter 02 & Catheter 03 \\
\hline $10-25 \mu \mathrm{m}$ & 206 & 235 & 138 \\
$25-50 \mu \mathrm{m}$ & 7 & 6 & 2 \\
$50-100 \mu \mathrm{m}$ & 0 & 1 & 0 \\
$100-500 \mu \mathrm{m}$ & 0 & 0 & 0 \\
\hline Total & 213 & 241 & 140 \\
\hline
\end{tabular}

Over $90 \%$ of the particles were found in the particle class $10-25 \mu \mathrm{m}$ for all tested solutions. Just one particle was found in the class of $50 \mu \mathrm{m}$ to $100 \mu \mathrm{m}$ and no particles larger than $100 \mu \mathrm{m}$ were detected.

\subsection{Particle counting and visualization with dynamic image analysis}

Representative images of the particles detected in the test suspension are shown in Figure 1. The particle edge trace is 


\section{DE GRUYTER}

A. Kurzhals et al., Dynamic image analysis of transparent particles released during the simulated use test of cardiovascular devices -205

drawn by the software along the particle border visualizing the different size detection for different threshold values.

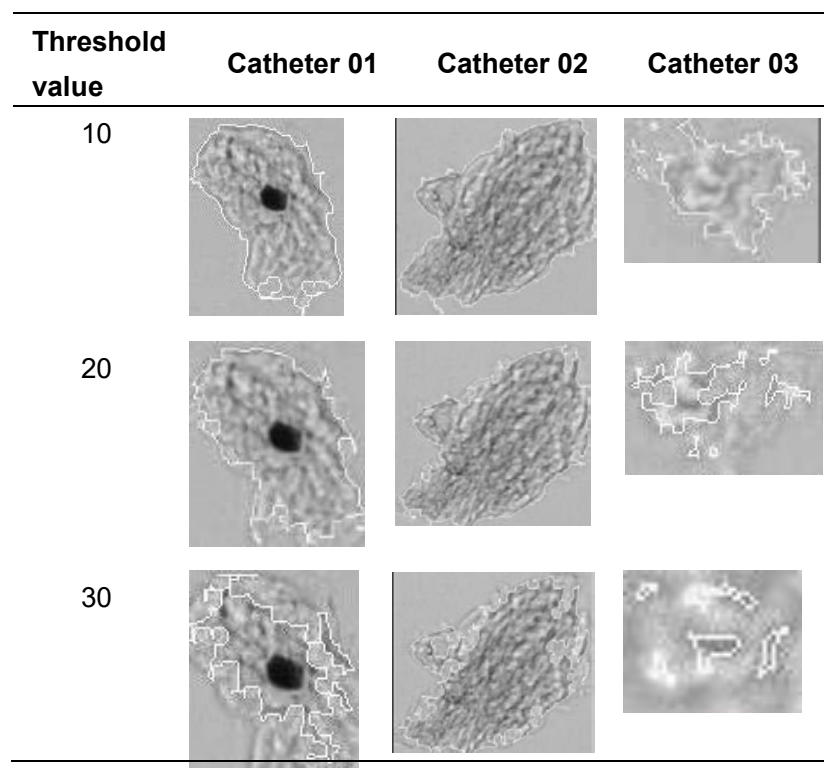

Figure 1: Percentage of particles in each size class of the total amount of particles, mean value of 3 test samples, used threshold values are given as 10,20 and 30

The results of the test suspension by DIA with different threshold values are given in table $2-4$. The results were calculated as particle concentration in particle per $\mathrm{ml}$.

Table 2: Particle concentration in particles per $\mathrm{ml}$ for a threshold value of 10

\begin{tabular}{rrrr}
\hline Particle size & Catheter 01 & Catheter 02 & Catheter 03 \\
\hline $10-25 \mu \mathrm{m}$ & 1489 & 1286 & 787 \\
$25-50 \mu \mathrm{m}$ & 573 & 484 & 274 \\
$50-100 \mu \mathrm{m}$ & 225 & 151 & 74 \\
$100-500 \mu \mathrm{m}$ & 46 & 27 & 13 \\
\hline Total & 2333 & 1948 & 1148 \\
\hline
\end{tabular}

Table 3: Particle concentration in particles per $\mathrm{ml}$ for a threshold value of 20

\begin{tabular}{rrrr}
\hline Particle size & Catheter 01 & Catheter 02 & Catheter 03 \\
\hline $10-25 \mu \mathrm{m}$ & 227 & 189 & 143 \\
$25-50 \mu \mathrm{m}$ & 98 & 66 & 52 \\
$50-100 \mu \mathrm{m}$ & 46 & 26 & 12 \\
$100-500 \mu \mathrm{m}$ & 17 & 14 & 12 \\
\hline Total & 388 & 295 & 219 \\
\hline
\end{tabular}

Table 4: Particle concentration in particles per $\mathrm{ml}$ for a threshold value of 30

\begin{tabular}{rrrr}
\hline Particle size & Catheter $\mathbf{0 1}$ & Catheter 02 & Catheter 03 \\
\hline $10-25 \mu \mathrm{m}$ & 124 & 89 & 88 \\
$25-50 \mu \mathrm{m}$ & 34 & 31 & 20 \\
$50-100 \mu \mathrm{m}$ & 6 & 10 & 8 \\
$100-500 \mu \mathrm{m}$ & 4 & 7 & 8 \\
\hline Total & 167 & 137 & 123 \\
\hline
\end{tabular}

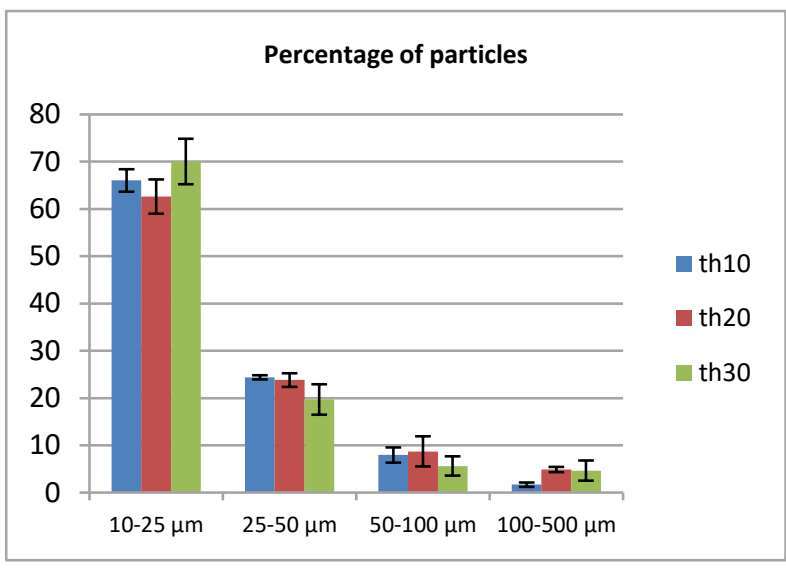

Figure 2: Percentage of particles in each size class of the total amount of particles, mean values and standard deviation $(n=3)$

Figure 2 shows the particle size distribution for three different threshold values. The percentage of particles in each size class of the total amount of particles was calculated for each test sample. The diagram shows the mean value of the three test samples. The size distribution is similar for different threshold values.

\section{Discussion}

The DIA with the FlowCam is well suited for taking images of transparent particles in suspension. Because of the backlighting they appear brighter than opaque particles. It is proposed that most of these particles were caused by the abrasion of the hydrophilic coating. The images show that the particle border meets the particle shape very well at threshold values of 10 and 20 in these examples. The determination of the diameter appears to be underestimated for a threshold value of 30 . The segmentation of particles worked for all thresholds. In case of higher concentration of the particles in suspension, particles could appear closer together in the images which could lead to under-segmentation. In this case, 
a reduction of the value "Distance nearest neighbour" can be of use [8].

Comparing the results of table 2 to table 4 it can be discerned that many of the particles detected with a threshold value of 10 were not even recognized as a particle with a threshold value of 20 or 30 . Another set of particles is detected, but the diameter is probably underestimated (smaller than $10 \mu \mathrm{m}$ ) so that those particles are not counted in the regarded particle classes. So the increase of the threshold value to 20 leads to a reduction of detected particles about $81-85 \%$ in this study and to a reduction about $89-93 \%$ for a threshold value of 30 . The determination of the total amount of particles in the test suspension is strongly depending on the threshold value. The relative particle size distribution is not influenced by the variation of the threshold value in this study. Figure 2 shows the percentage of particles in each class for the three threshold values.

As a result it can be seen that more particles were found with the DIA method than with the LOM when the greyscale threshold was set on a value of 10 or 20 . For a threshold value of 30 the total amount of particles is smaller compared to the results of the LOM counting.

Looking at the particle size distribution, the amount of particles with a diameter of $\geq 25 \mu \mathrm{m}$ is higher for all threshold values during DIA measurement than for the LOM counting. More than $30 \%$ of the particles were sized larger than $25 \mu \mathrm{m}$ for all threshold values with the DIA whereas less than $5 \%$ of the total amount of particles showed a larger diameter than $25 \mu \mathrm{m}$ with LOM. Between 5 and $16 \%$ of the particles per $\mathrm{ml}$ were found in the range of 50 to $500 \mu \mathrm{m}$ by DIA, while no particles of this dimension were counted by LOM. Hence, it can be assumed that transparent particles larger than $50 \mu \mathrm{m}$ are not detected completely by the detector of the LOM counter.

That the size of transparent particles is underestimated and the particle counts were lower with LOM was already found in other studies, where proteins were an example for transparent particles [9].

A limitation of the study is the different sample volume used in both methods, which could have an influence on the results. Also remarkable are the low particle concentrations.

\section{Conclusion}

The determination of the threshold value has a high influence on the particle counting and sizing. The amount of counted particles is higher for lower threshold values when the test suspension contains many transparent particles.
The comparison of the determined particle concentration shows that the results can differ for both methods.

Since the default threshold value is 20 it could be considered to lower the value for the analysis of samples that contain mainly transparent particles.

The DIA is a suitable method to evaluate the visual appearance of the particles in addition to the particle counts. The method shows particles larger than $25 \mu \mathrm{m}$ that could be overlooked with the LO method. It is well suited for the characterization of particle size distribution but not for the rating of the total particle counts because the results are depending on the measuring parameters.

\section{Author Statement}

Research funding: This work was partially funded by the Federal Ministry of Education and Research (BMBF) within the project RESPONSE "Partnership for Innovation in Implant Technology". Informed consent is not applicable. Ethical approval: The conducted research is not related to either human or animal use.

\section{References}

[1] Babcock DE, Hergenrother RW, Craig DA, Kolodgie FD, Virmani R. In vivo distribution of particulate matter from coated angioplasty balloon catheters. Biomaterials 2013; 34: 3196-3205

[2] ASTM F2743-11 (2018), Standard Guide for Coating Inspection and Acute Particulate Characterization of Coated Drug-Eluting Vascular Stent Systems, ASTM, 2018

[3] DIN EN ISO 25539-2 (2012): Cardiovascular implants Endovascular devices - Part 2: Vascular stents, 2012

[4] US Pharmacopeia: Particulate matter in injections. USP/NF General Chapter <USP 788>. In: U.S. Pharmacopeia, editor. Rockville, MD: USP; 2009. National Formulary, USP32-NF27 (Suppl. 2)

[5] ASTM F2394-07(2017), Standard Guide for Measuring Securement of Balloon Expandable Vascular Stent Mounted on Delivery System, ASTM, 2017

[6] Cao S, Narhi L, Jiang Y, Rajan RS. Analytical methods to measure subvisible particulates: Analysis of aggregates and particles in protein pharmaceuticals. John Wiley \& Sons, Inc., Hoboken, New Jersy, 2012. 85-115.

[7] A white paper:The Benefits of Light and Dark Pixel Thresholding, Fluid Imaging Technologies Inc, URL: https://info.fluidimaging.com/hubfs/whitepapers/The \%20Benefits\%20of\%20Light\%20and\%20Dark\%2 OPixel\%20Thresholding\%20Whitepaper.pdf (June 14th2019

[8] FlowCAM® Manual, Version 3.4, 2014, Fluid Imaging Technologies Inc.

[9] Werk T, Volin DB, Mahler HC. Effect of solution properties on the counting and sizing of subvisible particle standards as measured by light obscuration and digital imaging methods , European Journal of Pharmaceutical Sciences, 2014; 53:95108 